\title{
Controlling HIV in adolescents: solutions for engagement, access and retention
}

\author{
Anna de Waal ${ }^{1}$ \& Nitika Pant Pai ${ }^{*}, 1,2$ \\ ${ }^{1}$ CORE, Research Institute of McGill University Health Centre, QC, Canada \\ ${ }^{2}$ Divisions of Clinical Epidemiology \& Infectious Diseases, Department of Medicine, McGill University, QC, Canada \\ *Author for correspondence: Tel.: +1 5149341934 ext. 44729; nitika.pai@mcgill.ca
}

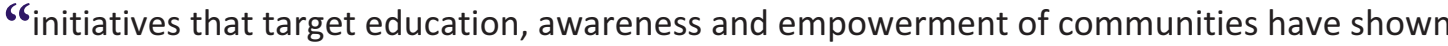 promise in engaging adolescents and young adults in HIV prevention"'}

First draft submitted: 26 March 2018; Accepted for publication: 25 April 2019; Published online: 8 July 2019

Keywords: access $\bullet$ adolescents $\bullet$ barriers $\bullet$ engagement $\bullet$ HIV $\bullet$ retention in care $\bullet$ testing

Adolescents and young adults across low- and middle-income countries bear a disproportionate burden of the global HIV epidemic and face specific barriers to engagement, access and retention in the HIV treatment cascade. An estimated 6000 new infections occur globally each day, and sub-Saharan Africa accounts for more than $70 \%$ of the burden of infection [1]. In this context, HIV prevention efforts targeting youth and adolescents between the ages of 15 and 24 is crucial in reducing HIV transmission and ensuring a productive workforce empowered to care for their family and elders. Lack of access to personalized HIV testing alongside interventions, which fail to adequately respond to the specific needs of adolescent populations, are salient barriers to entry into HIV care [2]. Pragmatic interventions that enhance engagement, access, linkages and continued retention in care need to be emphasized through the integration of multi-level strategies.

\section{Education, awareness \& empowerment of communities}

Community-based organizations are frontline actors that promote education regarding adolescent vulnerability to HIV to help them mediate their own risk. Emerging research on adaptable, multisectoral approaches that layer interventions across health, social welfare and education sectors has shown promise [3]. The DREAMS Partnership implemented in Kenya, South Africa and Zimbabwe has been well received in community settings and ensures community ownership by working with existing community-based organizations to layer multiple interventions as integrated 'packages' targeting HIV vulnerability [3]. In addition, a growing body of evidence supports the use of conditional cash transfers (CCTs) to reduce the risk of HIV acquisition in young women. As financial incentives conditional on school attendance, CCTs aim to reduce risky behaviors and thereby address the socioeconomic drivers that influence HIV transmission. The CCTs are feasible in endemic settings where monthly cash transfers may mitigate the role of age-disparate or intergenerational relationships, as young women resorting to transactional sexual relationships are identified as a key driver of the age-sex disparity in HIV acquisition in the sub-Saharan African context [4]. Stigma as a structural driver affecting entry to the HIV treatment and care cascade may be addressed by strategies that empower youth and adolescent populations to make autonomous decisions regarding testing and counseling. In sum, initiatives that target education, awareness and empowerment of communities have shown promise in engaging adolescents and young adults in HIV prevention.

\section{Access to HIV testing}

Literature on improving access to HIV testing for adolescents has focused on the use of novel HIV self testing (HIVST) interventions, understanding motivations for seeking testing and suggestions of self-testing linked to preexposure prophylaxis (PrEP) as emerging strategies require greater emphasis [5]. HIVST is a powerful alternative to conventional facility-based testing that could support the agency of adolescents to make them aware of their sero-status, while addressing structural barriers such as stigma, inconvenience and parental consent required for 
adolescent testing [6]. Literature on HIVST has shown a high acceptability in adolescent populations from countries such as Zambia [7], Mozambique [8] and South Africa [9].

Early oral PrEP and post-exposure prophylaxis further show promise as effective prevention interventions that may inhibit HIV acquisition in youth populations, averting future costs of treatment [10]. Research in adult discordant populations in Uganda and Kenya found those taking oral tenofovir or tenofovir plus emtricitibine (Truvada) as prophylaxis had a reduced HIV acquisition rate of 67 and $75 \%$ respectively [11]. However, literature supporting its use in adolescent populations is sparse. While the effectiveness of PrEP is supported by an increasing number of randomized, controlled trials, greater availability of PrEP will help improve its uptake and prevent infections in adolescents in sub-Saharan Africa and Southern Africa [12]. It will further increase the uptake of HIVST and reduce rates of transmission.

\section{Retention}

Despite persistent challenges in linkage to care for adolescents diagnosed with HIV, a lack of research exists surrounding interventions specific to this population. A systematic review and meta-analysis of the adolescent HIV care continuum in South Africa found significantly lower retention in care and viral suppression rates for adolescents compared with adults [13]. Although evidence has supported the delivery of community-based services and the decentralization of health services to improve linkage to care, its widespread implementation has been minimal. In a study among adolescents aged 16 and older in Malawi, HIV-positive patients were assigned to facility-based care or optional home initiation of HIV care; participants who chose home initiation of HIV care had a 2.44-fold greater rate of antiretroviral therapy (ART) initiation compared with those in the facility group, suggesting that the availability of home initiation of HIV care could remove barriers including mistrust of clinicbased services, fear of stigma and disclosure and logistic barriers such as transportation costs [14]. Parental consent, parental engagement and counseling services for young adults will be key to operationalizing these services in these populations, on a continued basis.

Electronic health (ehealth) and mobile health (mhealth) interventions have demonstrated efficacy and effectiveness in improving ART adherence among adolescent populations [15]. Interventions delivered by SMS/texting, smartphone apps or social media campaigns that capitalize upon smartphone prevalence and use of mobile devices are yet to be untapped for scale-up. Including young adult populations in the design of technology-based interventions is critical to ensuring that clinical and psychosocial needs, impacting all levels of care from access and engagement to linkage and retention, are met [16].

\section{Conclusion}

Attaining the UNAIDS goal of an 'AIDS-free generation' critically depends upon addressing the specific needs of adolescents and young to reduce HIV transmission. While many of the barriers to HIV testing in sub-Saharan youth are similar to those faced by adults, adolescents are more dependent on family and as such require greater support in decision-making and look to adults and their communities for guidance. The context of adolescent risk behaviors must be addressed by community-wide educational campaigns and media interventions that promote an open discourse among family members and peers surrounding HIV transmission and prevention. Youth-focused engagement strategies including mhealth interventions and community-based personalized services tailored to their unique socio-environmental context will improve engagement in the gamut of services from HIV testing, to PrEP and ART adherence, to retention in care to prevent transmission. Along with research, impactful interventions that demonstrate success are the need of the hour. Scaling up proven interventions will generate hope and minimize the brunt of the HIV epidemic in adolescent and young adult populations across low- and middle-income countries.

\section{Financial \& competing interests disclosure}

This work was supported by operating grants from the Canadian Institutes of Health Research (grant numbers HPP-137872 and PJT153149), Grand Challenges Canada Stars in Global Health grants (grant numbers 0732-05 and 0710-05) and the FRQS ResearchScholar Senior awarded to Nitika Pant Pai. The authors have no other relevant affiliations or financial involvement with any organization or entity with a financial interest in or financial conflict with the subject matter or materials discussed in the manuscript apart from those disclosed.

No writing assistance was utilized in the production of this manuscript. 


\section{Open access}

This work is licensed under the Attribution-NonCommercial-NoDerivatives 4.0 Unported License. To view a copy of this license, visit http://creativecommons.org/licenses/by-nc-nd/4.0/

\section{References}

1. Kharsany AB, Karim QA. HIV infection and AIDS in sub-Saharan Africa: current status, challenges and opportunities. Open AIDS J. 10, 34-48 (2016).

2. Kurth AE, Lally MA, Choko AT, Inwani IW, Fortenberry JD. HIV testing and linkage to services for youth. J. Int. AIDS Soc. 18(2 Suppl. 1), 19433-19433 (2015).

3. Chimbindi N, Birdthistle I, Shahmanesh M et al. Translating DREAMS into practice: early lessons from implementation in six settings. PLoS ONE 13(12), e0208243 (2018).

4. Wamoyi J, Stobeanau K, Bobrova N, Abramsky T, Watts C. Transactional sex and risk for HIV infection in sub-Saharan Africa: a systematic review and meta-analysis. J. Int. AIDS Soc. 19(1), 20992 (2016).

5. Bekker L-G, Hosek S. HIV and adolescents: focus on young key populations. J. Int. AIDS Soc. 18(2 Suppl. 1), 20076 (2015).

6. Zanoni BC, Elliott RJ, Neilan AM, Haberer JE. Screening for HIV and linkage to care in adolescents: insights from a systematic review of recent interventions in high- versus low- and middle-income settings. Adolesc. Health Med. Ther. 9, 211-235 (2018).

7. Zanolini A, Chipungu J, Vinikoor MJ et al. HIV self-testing in Lusaka Province, Zambia: acceptability, comprehension of testing instructions, and individual preferences for self-test kit distribution in a population-based sample of adolescents and adults. AIDS Res. Hum. Retroviruses 34(3), 254-260 (2018).

8. Hector J, Davies MA, Dekker-Boersema J et al. Acceptability and performance of a directly assisted oral HIV self-testing intervention in adolescents in rural Mozambique. PLoS ONE 13(4), e0195391 (2018).

9. Smith P, Wallace M, Bekker LG. Adolescents' experience of a rapid HIV self-testing device in youth-friendly clinic settings in Cape Town South Africa: a cross-sectional community based usability study. J. Int. AIDS Soc. 19(1), 21111 (2016).

10. Baggaley R, Dalal S, Johnson C et al. Beyond the 90-90-90: refocusing HIV prevention as part of the global HIV response. J. Int. AIDS Soc. 19(1), 21348-21348 (2016).

11. Baeten JM, Donnell D, Ndase P et al. Antiretroviral prophylaxis for HIV prevention in heterosexual men and women. N. Engl. J. Med. 367(5), 399-410 (2012).

12. Dellar RC, Dlamini S, Karim QA. Adolescent girls and young women: key populations for HIV epidemic control. J. Int. AIDS Soc. 18(2 Suppl. 1), 19408-19408 (2015).

13. Zanoni BC, Archary M, Buchan S, Katz IT, Haberer JE. Systematic review and meta-analysis of the adolescent HIV continuum of care in South Africa: the Cresting Wave. BMJ Glob. Health 1(3), e000004 (2016).

14. Mavegam BO, Pharr JR, Cruz P, Ezeanolue EE. Effective interventions to improve young adults' linkage to HIV care in Sub-Saharan Africa: a systematic review. AIDS Care 29(10), 1198-1204 (2017).

15. Sayegh CS, Macdonell KK, Clark LF et al. The impact of cell phone support on psychosocial outcomes for youth living with HIV nonadherent to antiretroviral therapy. AIDS Behav. 22(10), 3357-3362 (2018).

16. Mulawa MI, Legrand S, Hightow-Weidman LB. eHealth to enhance treatment adherence among youth living with HIV. Curr. HIV/AIDS Rep. 15(4), 336-349 (2018). 
